\title{
Desastre da Vale: o desafio do cuidado em Saúde Mental e Atenção Psicossocial no SUS
}

\author{
The disaster of Vale in Brazil: Mental Health and Psychosocial Care \\ challenges for SUS
}

Débora da Silva Noal', Vanuse Maria Resende Braga ${ }^{\mathbf{2}}$, Mariana Bertol Leal ${ }^{\mathbf{3}}$, Angela Ribeiro Vargas ${ }^{3}$, Paula Eliazar ${ }^{3}$

DOI: 10.1590/0103-11042020E224

RESUMO Em 25 de janeiro de 2019, a barragem Córrego do Feijão da empresa Vale S.A., em Brumadinho, Minas Gerais, rompeu-se atingindo a área administrativa da empresa e a área rural do entorno, deixando corpos, capacidade produtiva e de subsistência, memórias e trajetórias sob a lama de rejeito de minérios. $\mathrm{O}$ rompimento da barragem desencadeou a morte direta de 249 pessoas e deixou 137 pessoas desabrigadas. Este artigo teve como objetivo relatar a experiência da implementação da estratégia de Saúde Mental e Atenção Psicossocial no Sistema Único de Saúde (SUS), considerando os três entes federados, bem como os atores que compuseram a estratégia desde as primeiras horas após o desastre até o final dos primeiros seis meses. Muitos foram os desafios para que o SUS se readaptasse à nova conjuntura, particularmente pela combinação dos seguintes fatores: sofrimento intenso e amplitude das perdas socioafetivas vivenciadas; escassez de emprego e recursos financeiros; consequências ambientais ainda pouco mensuradas; e demanda por ressignificação de uma identidade da comunidade residente. Um trabalho articulado entre o SUS e os diferentes setores, como a educação e a assistência social, mostrou-se importante e primordial, indicando ser necessária a continuidade desses pontos na agenda do SUS.

PALAVRAS-CHAVE Desastres. Saúde mental. Sistemas de apoio psicossocial. Saúde pública.

1Fundação Oswaldo Cruz (Fiocruz), Centro de Estudos e Pesquisas em Emergências e Desastres em Saúde (Cepedes) - Rio de Janeiro (RJ), Brasil. noaldebora@gmail.com

2 Secretaria Municipal de Saúde de Brumadinho (SMSB) - Brumadinho (MG), Brasil.

${ }^{3}$ Ministério da Saúde (MS) - Brasília (DF), Brasil.
ABSTRACT On January 25, 2019, the Córrego do Feijão dam of Vale SA, in the city of Brumadinho, Minas Gerais, broke down reaching the company's administrative area and the surrounding rural area, leaving bodies, productive and subsistence capacity, memories and trajectories under the sludge of ore tailings. The rupture of the dam triggered the direct death of 249 people and left 137 people homeless. This article aimed to report the experience of implementing the strategy of Mental Health and Psychosocial Care in the Unified Health System (SUS), considering the three federated entities, as well as the actors that composed the strategy from the first hours after the disaster until the end of the first six months. There were many challenges for the SUS to adapt to the new situation, particularly due to the combination of the following factors: intense suffering and the extent of the socio-affective losses experienced; scarcity of jobs and financial resources; environmental consequences still poorly measured; and demand for the resignification of an identity of the resident community. Articulated work between the SUS and the different sectors, such as education and social assistance, proved to be important and primordial, indicating that it is necessary to continue these points on the SUS agenda.

KEYWORDS Disasters. Mental health. Psychosocial support systems. Public health. 


\section{Introdução}

Este artigo é um relato de experiência, escrito a partir da reflexão das autoras, após o rompimento da barragem em Brumadinho, onde participaram das fases de resposta e recuperação. Respectivamente, as autoras trabalharam como: membro de uma organização internacional de ajuda humanitária especialista em desastres convidada a integrar o Centro de Operações de Emergências em Saúde (Coes) na primeira fase do desastre, membro da gestão municipal da saúde de Brumadinho e membros da gestão federal do Sistema Único de Saúde (SUS), especificamente, a Força Nacional do SUS.

A barragem I da Mina do Córrego do Feijão da empresa Vale S.A., em Brumadinho, no estado de Minas Gerais, rompeu no dia 25 de janeiro de 2019, atingindo a área administrativa da empresa (alojamento/restaurante) e a área rural do entorno, deixando sob a lama de rejeito de minérios: corpos, capacidade produtiva e de subsistência, memórias e trajetórias. No momento do desastre, Brumadinho possuía uma população estimada em 40.103 habitantes distribuídos em uma extensão territorial de $639,434 \mathrm{~km}^{2}$.

Este foi classificado como o maior acidente ampliado de trabalho no Brasil e ocorreu há 25 dias da mudança na gestão dos governos federal e estadual. Não foi uma tragédia anunciada, embora fosse possível de ser prevista. Esse desastre desencadeou consequências para a vida daquele município, para a região e, consequentemente, para Minas Gerais e para o Brasil.

O rompimento da barragem desencadeou a morte direta de 249 pessoas e deixou 137 pessoas desabrigadas, segundo informações do Boletim Estadual de Proteção e Defesa Civil de Minas Gerais, publicado em 25 de setembro de $2019^{2}$. Seis meses após o desastre, 21 pessoas continuavam desaparecidas, potencializando a instabilidade emocional e complexificando o processo de enlutamento das famílias afetadas ${ }^{3}$.

No que concerne às especificidades do território afetado, nos seis meses subsequentes ao desastre, os profissionais das Equipes Intermediárias de Saúde Mental (EISM) de Brumadinho, criadas para implementar as ações de cuidado à população afetada pelo desastre, observaram que, com o aumento do fluxo de pessoas na cidade, os munícipes passaram a se referir a essa população externa como 'forasteiros'. Com esse termo, reportavam-se àqueles que não residiam antes do desastre e que chegaram nas fases de resposta e reconstrução e, por ali, encontraram uma 'oportunidade' de vida segundo a população. Embora tenha aumentado o número de trabalhadores oriundos de outras cidades, percebeu-se, pela equipe gestora do SUS municipal, a necessidade de um contingente ainda maior de profissionais, tendo em vista o aumento da população, dos problemas e dos adoecimentos desencadeados pelo evento.

Parte da narrativa manifestada pela população durante os atendimentos de saúde mental da rede municipal de saúde referia-se ao rápido aumento populacional: "essa cidade não é mais a mesma", "a gente sai na rua e não conhece mais ninguém". As causas desse aumento populacional frequentemente eram atribuídas aos depósitos indenizatórios realizados pela empresa causadora do rompimento da barragem na conta da população que comprovasse residência, no período anterior ao desastre ou que morasse até $1 \mathrm{~km}$ da calha do Rio Paraopeba desde Brumadinho até a cidade de Pompéu, no dia 25 de janeiro de 2019. Tal comprovação deveria se dar por meio de registros e declarações de endereços oriundas de escolas, faculdades, Centro de Referência de Assistência Social (Cras), entre outras instituições públicas como as unidades de saúde ${ }^{4}$.

Parte das narrativas de desconforto acima descritas estavam vinculadas ao sofrimento acarretado pela alteração da estrutura socioafetiva e geográfica da cidade. Na perspectiva da população: "a cidade não é mais a mesma, $e$ talvez nunca mais vai ser", na medida em que aquela cidade onde haviam sido edificadas histórias, sonhos e planos já não existia mais, demandando a necessidade de elaborar o "luto pela cidade perdida". 
Esse aumento da população, em decorrência do desastre, acarretou conflitos entre residentes e 'forasteiros' que discutiam a legitimidade do recebimento de tal indenização por pessoas que, segundo eles, não contemplavam os pré-requisitos exigidos pela empresa causadora do desastre. As equipes de saúde, por sua vez, manifestaram insatisfação pela grande sobrecarga de serviço adicional no atendimento direto, bem como pela pressão sofrida para fornecerem as declarações de residência solicitadas. Em decorrência dessas demandas adicionais, parte das ações e programas rotineiros do cuidado no âmbito da atenção primária não foram desenvolvidas nesse período pós-desastre, deixando a assistência parcialmente comprometida, o que poderá será observado nos indicadores de produtividade.

Houve mudanças bruscas de hábitos e rotinas dos munícipes, impactando a saúde mental da população, provocando um aumento das buscas por atendimento, alterações das condições de vida e de saúde, principalmente de saúde mental, como é possível acompanhar no gráfico $1^{5}$. Além do aumento da gravidade dos casos de saúde mental e condições clínicas já existentes, bem como do surgimento de novos casos a partir do momento em que passaram a experienciar o período de luto, foi necessário acolher o sofrimento da população pela mudança de rotina da cidade.

Gráfico 1. Atendimentos das equipes especializadas em saúde mental

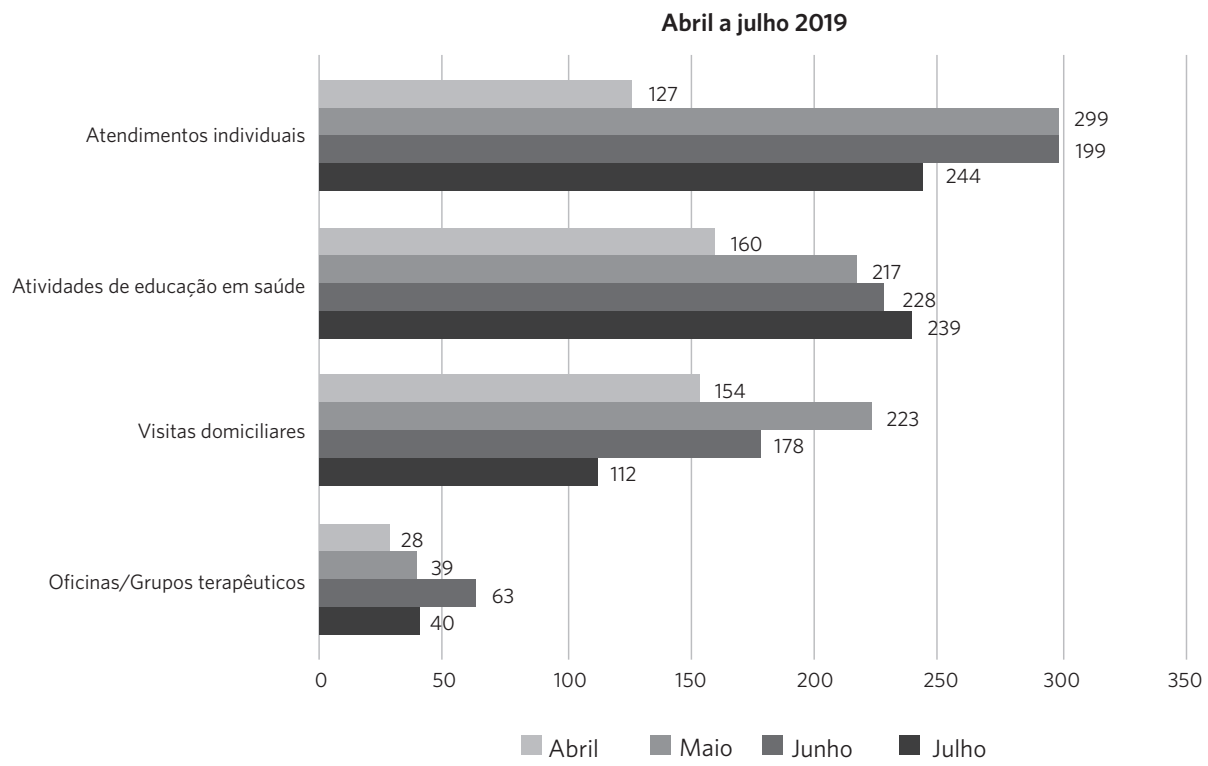

Fonte: Sistema E\&L da Prefeitura de Brumadinho5.

Ainda no que concerne ao impacto direto causado pelo rompimento da barragem, cabe lembrar que a principal atividade econômica do município de Brumadinho e região, no momento do desastre, era a mineração, o que contribuiu para que o impacto sociopolítico, cultural e econômico ganhasse uma maior amplitude. $\mathrm{O}$ desastre também afetou parte da economia do município que tinha como lastro o turismo, particularmente o ecoturismo e as visitas a um museu de reconhecimento internacional com um significativo acervo de 
arte contemporânea a céu aberto ${ }^{6}$, causando apreensão aos moradores que tinham no turismo sua fonte de renda, bem como temiam deixar de ser residentes em uma rota turística e passassem a ser identificados como moradores da 'cidade do desastre', condenando-os à repetição das memórias de sofrimento.

\section{A rede de cuidados e suas ressignificações}

A rede de saúde do município de Brumadinho, no momento do desastre, tinha como desenho organizador da assistência a Estratégia Saúde da Família (ESF), com 17 equipes distribuídas em 14 Unidades Básicas de Saúde (UBS), e uma cobertura territorial de atenção primária de $100 \%$. As equipes eram compostas por médico, enfermeiro, técnico de enfermagem e agentes comunitários; e, em algumas delas, equipes ampliadas contavam com odontólogos e técnicos de saúde bucal.

Ainda que a cobertura da atenção primária fosse ampla, a extensão territorial do município dificultava o acesso de parte dos usuários a rede do SUS; por essa razão, a rede contava ainda com 22 pontos de apoio para as equipes da ESF, dois deles com equipes compostas por: enfermeiros, técnicos de enfermagem, agentes comunitários, médicos generalistas e psiquiatras alguns dias por semana.

De forma complementar ao trabalho das equipes das UBS havia duas equipes do Núcleo de Apoio à Saúde da Família (Nasf), compostas por: psicólogas, fonoaudiólogas, educador físico, terapeuta ocupacional, fisioterapeutas, assistentes sociais e nutricionistas. No momento do desastre, o município contava ainda com uma Unidade de Pronto Atendimento 24 horas (UPA), uma Unidade de Atendimento Imediato na zona rural (UAI - Piedade) que funcionava à noite e nos finais de semana e feriados, um hospital - que atendia os encaminhamentos da UPA, cirurgias eletivas e urgência para partos e cesáreas -, uma policlínica com as seguintes especialidades: clínica geral, cardiologia, dermatologia, fonoaudiologia, ortopedia, ginecologia, geriatria, hematologia, ortopedia, pediatria, reumatologia e urologia. A rede era composta ainda por um Centro de Reabilitação com fisioterapeutas e terapeutas ocupacionais e um Núcleo de Práticas Integrativas e Complementares em Saúde (Nupic) com as seguintes práticas: medicina tradicional chinesa, acupuntura, auriculoterapia, reiki, arteterapia, terapia floral, massoterapia, terapia de florais, aromaterapia e cromoterapia.

A Rede de Atenção Psicossocial (Raps) do município, por sua vez, contava com um Centro de Atenção Psicossocial (Caps II), um Centro de Atenção Psicossocial Infanto-juvenil (Capsi), um ambulatório para o cuidado a usuários de álcool e outras drogas que funcionava dentro do Caps II, e quatro leitos psiquiátricos no Hospital Geral. Frisa-se que a amplitude de serviços foi fundamental para a elaboração de uma estratégia de cuidado pós-desastre rápida e consistente, tendo como base os princípios e diretrizes do SUS.

Como um dos pontos prioritários da implementação da estratégia de Saúde Mental e Atenção Psicossocial (SMAPS) na primeira fase do desastre, sensibilizaram-se os trabalhadores do SUS, por meio de capacitações em cada um dos dispositivos de saúde, haja vista que, em situações recentes de desastres, as reações psicossociais, até o fim do primeiro trimestre, são consideradas esperadas para uma situação que extrapola a rotina. Dessa forma, informando que a população que padece de sofrimento agudo não necessariamente tem ou terá um transtorno de saúde mental em médio ou longo prazos ${ }^{7,8}$, como pode ser visto no quadro 1 .

Enfatiza-se que, embora estatisticamente o número de transtornos não seja muito maior que o existente no período pré-desastre (ver quadro 1), o aumento no número de casos aumenta consideravelmente quando as ações e as estratégias de atenção psicossocial, particularmente os primeiros auxílios psicológicos ${ }^{9}$, incluindo a psicoeducação e a orientação concernente às reações 'normais' pós-desastre, não são implementadas na primeira fase do desastre ${ }^{10}$. 
Quadro 1. Impacto dos desastres na morbidade por transtornos mentais

\begin{tabular}{lll}
\hline Transtornos mentais e reações ao estresse & $\begin{array}{l}\text { Antes do desastre } \\
\text { Prevalência-12 meses }\end{array}$ & $\begin{array}{l}\text { Depois do desastre } \\
\text { Prevalência-12 meses }\end{array}$ \\
\hline $\begin{array}{l}\text { Transtornos mentais severos } \\
\text { (ex.: psicoses, depressão severa, transtornos } \\
\text { de ansiedade gravemente incapacitantes) }\end{array}$ & $2 \%-3 \%$ & $3 \%-4 \%$ \\
$\begin{array}{l}\text { Transtornos mentais leves ou moderados } \\
\text { (ex.: transtornos de depressão e ansiedade } \\
\text { leve ou moderados) }\end{array}$ & $10 \%$ & $15 \%-20 \%$ (reduzem com o tempo) \\
$\begin{array}{l}\text { Reações de estresse 'normal' (sem trans- } \\
\text { torno) }\end{array}$ & Não há uma estimativa conclusiva & $\begin{array}{l}\text { \% alta que tende a reduzir subs- } \\
\text { tancialmente com o tempo }\end{array}$ \\
\hline
\end{tabular}

Fonte: $\operatorname{lasc}^{8}$

\section{Reconfigurações da rede municipal pós-desastre}

Considerando a abrangência da demanda emergencial e a cobertura alcançada pela rede municipal de saúde, parte do apoio dos entes federados, particularmente do Ministério da Saúde e da Secretaria Estadual de Saúde, voltaram-se para a realização de uma avaliação com as equipes do município a fim de estabelecer de forma pactuada a estratégia mais compatível com a reorganização da rede assistencial, considerando o perfil das demandas, a partir da capacidade instalada pré-desastre.

Definiu-se que, em decorrência do impacto psicossocial pós-desastre, da agudização de casos já estáveis e da tendência de aumento no número de casos em médio e longo prazos, haveria a ampliação da rede de atenção à saúde, principalmente a Raps, conforme já apresentado no gráfico $1^{5}$. O Capsi foi ampliado, e sua habilitação mudou para tipologia II; o Capsi, por sua vez, foi habilitado e passou a contar com recursos federais de custeio. Ainda em decorrência da previsão de aumento da demanda, foram habilitadas três equipes multiprofissionais de saúde mental para ampliar o acesso e potencializar a articulação da rede, as EISM.

Tais equipes implantadas pela gestão municipal foram ancoradas na Nota Técnica $n^{0} 11 / 2019^{11}$, que tem por objetivo prestar tratamento multiprofissional em saúde mental, integrando-se aos demais serviços da Raps e das redes de atenção à saúde. Optou-se pela implementação dessas equipes tendo em vista a necessidade de ampliar as estratégias de cuidado de forma integrada à atenção básica e à rede especializada. Tal dispositivo prestou-se ainda a contemplar o atendimento da demanda reprimida, posicionando-se como equipe intermediária entre os Caps e os dispositivos da atenção primária.

Outro recurso implementado na estratégia foi o ambulatório de saúde mental, estruturado na mesma área física do recém-habilitado Caps II, com o objetivo de acompanhar os pacientes estabilizados que ainda necessitavam de um serviço de referência com profissionais aptos para a continuidade do cuidado, evitando que as equipes da atenção primária fossem sobrecarregadas com casos que extrapolavam a complexidade da sua alçada. Para fortalecer o desenho de rede recém-reconfigurada, a implementação das ações de atenção psicossocial, de forma articulada com a ESF, mostrou-se imprescindível ao processo de cuidado.

A estratégia de cuidado para a SMAPS municipal pós-desastre possibilitou uma integração entre os dispositivos de saúde já existentes na rede municipal, propiciando uma ampliação do cuidado não apenas georreferenciado, mas uma equipe de cuidadores com conhecimento 
da situação da SMAPS anterior ao período do desastre, agilizando e facilitando o vínculo e o cuidado singular para os afetados, bem como proporcionando uma maior agilidade e precisão na identificação da população mais afetada pelo evento.

Ainda no que concerne ao fato da estratégia pós-desastre estar ancorada na rede de saúde georreferenciada já existente, ressaltamos que a percebemos como mais um fator de estímulo à confiança dos afetados nas equipes de saúde e, consequentemente, na adesão aos projetos terapêuticos desenvolvidos por estas, tendendo a proporcionar, também, uma maior responsabilização das equipes de atenção primária aos casos de saúde mental. A potência da ESF oportunizou um cuidado mais próximo ao usuário e a sua rede de apoio e suporte familiar ou social.

Ao acompanharmos os primeiros meses pós-desastre em Brumadinho, é possível perceber a imprescindibilidade de que os casos de SMAPS não sejam de cuidado exclusivo de um único serviço ou rede. Entendemos que o cuidado multiprofissional ampliado, particularmente no território onde o usuário se sente confortável e acolhido, oferece uma maior precisão e rapidez no processo de vinculação e reconhecimento do sofrimento, uma vez que esses já têm familiaridade e confiança.

Um ponto significativo de análise versa sobre os receios manifestados por profissionais da ESF em atender pacientes em sofrimento agudo pós-desastre, parte desses por não se sentirem capacitados para o manejo clínico dos casos. Nesses termos, o planejamento das ações de manejo clínico, atrelado às orientações referentes aos primeiros cuidados psicossociais pós-desastre, assim como a discussão de temas pertinentes à prática do cuidado ao portador de sofrimento psíquico agudo, não apenas fortalece o cuidado, mas oferece aos profissionais a segurança para o manejo clínico, oportunizando a construção de uma rede sólida e acessível, garantindo ao usuário o cuidado em diversos pontos da rede, conforme seu estado clínico.
No âmbito das políticas públicas nacionais, a articulação de ações de SMAPS na atenção primária tem sido preconizada pelo Ministério da Saúde. Nas últimas décadas, foram publicados documentos que estimulam essa articulação e apontam como estratégias o apoio matricial de saúde mental às equipes da atenção primária e a priorização da SMAPS na formação das equipes de saúde ${ }^{12}$.

Assim, foi objetivo da estratégia da SMAPS, de forma conjunta com os processos de reorganização da rede de atenção à saúde, a sensibilização dos profissionais assim como movimentos de produção de encontros entre profissionais e serviços para que pudessem ser produzidos laços e redes. Dessa forma, foram oportunizadas possibilidades efetivas de articulação para um cuidado continuado e ampliado, como, por exemplo, a ampliação e ressignificação da rede assistencial do município, especialmente os serviços e ações voltadas para SMAPS.

Lembramos que, embora o desastre tenha promovido um aumento na procura por atendimento nos dispositivos de cuidado do SUS, identificou-se que o evento potencializou a organização dos serviços de saúde e das estratégias de cuidado, ampliando a possibilidade de contribuir para a melhor resolutividade das situações no nível local13.

Vale pontuar que, considerando a rotina e a demanda existente no município no período pré-desastre, a rede assistencial já era estruturada e resolutiva para a população com o arranjo de vida existente antes do rompimento da barragem. Entretanto, com as novas urgências, surgiu a necessidade de uma ressignificação potencializando uma maior integração dos dispositivos da rede, particularmente da ESF com as equipes do Nupic e do Caps, e as novas equipes de matriciamento de SMAPS criadas especificamente para cuidar da população afetada pelo desastre da Vale S.A. no município. Tais equipes incorporaram em suas atividades a discussão de casos e articulações para um acompanhamento mais amplo e singularizado, estabelecendo a referência e a 
contrarreferência do modo não hierarquizado, responsável e implicado.

Outro aspecto a ser considerado foi a presença massiva de atores locais, regionais, nacionais e internacionais. A ampla participação desses atores externos foi intensificada nos primeiros 60 dias pós-desastre. Tais atores atuaram ativamente para que essas ofertas de cuidado e esse processo de reorganização da rede assistencial do SUS contemplassem a demanda emergencial. Nesses termos, a coordenação central desses atores, capitaneada pelo Coes, foi fundamental para que as ofertas fossem complementares e integradas à rede municipal de saúde já existente.

O Coes, composto por um grupo de gestores - entre eles, o secretário municipal adjunto de saúde, coordenadores da atenção primária, vigilância sanitária, saúde mental, urgência e emergência, além de referências técnicas e profissionais pertinentes às áreas da Secretaria Estadual de Saúde, bem como os técnicos da Força Nacional do SUS (FN-SUS) -, foi o responsável pela estrutura organizacional da estratégia com o objetivo de promover a resposta coordenada por meio da articulação e da integração dos atores envolvidos.

O Coes possibilitou uma maior precisão na análise dos dados e informações, com o intuito de subsidiar a tomada de decisão dos gestores e técnicos, na definição de estratégias e ações adequadas e oportunas para o enfrentamento de emergências em saúde pública. Com o auxílio do Coes, foram elaborados planos de contingência de acordo com cada uma das fases iniciais de resposta, isto é, para os primeiros 7 dias, 30 dias, 180 dias e, posteriormente, um de médio e longo prazo, com o objetivo de preparar o setor saúde para a fase de reconstrução.

Como um dos atores centrais do Coes, a FN-SUS permaneceu em Brumadinho nos primeiros 30 dias da fase inicial de resposta, apoiando as ações de reorganização da rede. De forma concomitante ao Coes municipal, foi implantado ainda um Coes nacional, que funcionou nos primeiros 47 dias pós-desastre.
Este tinha como objetivo apoiar as ações locorregionais a partir da articulação com os demais atores do nível central.

Após esse primeiro período, as estratégias de apoio entre as esferas do SUS foram sendo fortalecidas por meio de reuniões e oficinas que continuaram sendo realizadas de acordo com o previsto nos planos iniciais. Esse modelo de resposta para situações de emergência mostrou-se fundamental tanto para o setor saúde como para a articulação com os demais setores respondedores.

\section{Estratégias de cuidado}

Tendo em vista que parte dos impactos percebidos na população de Brumadinho versava sobre as necessidades de ressignificarem seus projetos de vida, crenças e valores, as equipes dos SUS foram estimuladas a pensar em estratégias que oportunizassem o cuidado de forma criativa, pragmática, não invasiva e sem forçar o usuário a falar ${ }^{\mathbf{1 4}}$.

Para a implementação da estratégia de cuidado, considerou-se que não apenas as famílias que perderam entes queridos foram afetadas, mas a totalidade dos munícipes sofrera o impacto psicossocial do desastre em escalas e intensidades distintas. As diferentes perdas despertam processos díspares de enlutamento e sofrimento.

Ofertas e abordagens de cuidado adaptadas às demandas precisaram ser organizadas, exigindo criatividade e flexibilidade da rede municipal de saúde. Foi necessário refletir sobre essa nova reorganização socioafetiva, bem como sobre os novos laços, identidades culturais e sociais a serem ressignificados. Essa foi uma ampla frente de trabalho das equipes de saúde do município com apoio de outras esferas do SUS assim como de parceiros nacionais e internacionais ${ }^{\mathbf{1 4}}$.

Um exemplo dessa oferta foi a ampliação da rede de cuidados referentes às práticas alternativas, que já existiam no município e que foram rapidamente acessadas e acolhidas pela população e, particularmente, pelas equipes 
de saúde do município. Embora fossem oferecidos aos profissionais de saúde do município diferentes formas de cuidado à SMAPS, como psicoeducação, escuta grupal e individual, a oferta de práticas integrativas, a partir do Nupic, foi rapidamente aceita e amplamente demandada pelos trabalhadores, particularmente da atenção primária.

Tendo como objetivo a oferta de tratamentos que utilizam recursos terapêuticos baseados em conhecimentos tradicionais, voltados para prevenir o aparecimento de doenças, os cuidados paliativos e determinadas doenças crônicas ${ }^{15}$, a equipe do Nupic disponibilizou, por meio do SUS municipal principalmente, as práticas de: medicina tradicional chinesa acupuntura, terapia de florais, massoterapia e reiki. Essa equipe foi intensamente solicitada pelos trabalhadores da rede municipal que rapidamente passaram a demandar visitas sistemáticas das equipes do Nupic em seus dispositivos de saúde, ampliando a oferta e a demanda para essa estratégia de cuidado singularizado.

Tendo em vista o aumento na demanda, foi necessário ampliar a capacidade de oferta, sendo que, em um primeiro momento, contou-se com voluntários e apoio de outras equipes do estado de Minas Gerais que se deslocaram para Brumadinho com o intuito de reforçar a rede. Logo após, foi possível a ampliação institucional da equipe do Nupic, propiciando que fosse possível ofertar um cuidado ainda mais ampliado aos usuários do serviço.

\section{A ampliação das equipes}

Já na primeira fase da resposta, mostrou-se necessária a contratação de uma nova equipe de atenção psicossocial específica para oferecer um suporte de SMAPS aos usuários na atenção primária. Foi preciso, inicialmente, acolher as demandas dos profissionais das equipes ESF e, na sequência, receber os casos concernente à SMAPS e levá-los para a discussão de reunião de matriciamento de forma gradativa, a fim de que a nova estratégia não fosse entendida como uma nova demanda para a atenção primária, em um cotidiano já repleto de ações e de responsabilidades.

Ainda que o suporte psicossocial aos usuários em fase anterior ao desastre já fosse parte das atribuições das ESF, em muitos casos, não fazia parte da rotina das equipes esse acompanhamento. Frisa-se que, passado o momento nevrálgico da emissão das declarações de comprovante de residências para aqueles que solicitaram a 'ajuda emergencial' da empresa causadora do desastre, passou-se a retomar as discussões sobre acolhimento psicossocial, fomentando a prática com a discussão de casos clínicos e, também, com temas pertinentes à prática, como o que é matriciamento, reações normais pós-desastre, suicídio e outros temas de interesses das equipes de ESF.

Fornecer às equipes informações sobre os temas recorrentes da SMAPS promoveu o fortalecimento da prática cotidiana, possibilitando às equipes de ESF uma melhor gestão dos casos, segurança na condução de algumas ações e, ao mesmo tempo, propiciando que as equipes de saúde mental priorizassem os casos mais graves e moderados, que demandam um acompanhamento mais especializado, garantindo cuidado integral aos portadores de sofrimento psíquico.

Ressalta-se que as equipes intermediárias, responsáveis pela condução das reuniões de matriciamento juntamente com os profissionais da atenção primária, têm como um dos objetivos principais ampliar o olhar e permitir acolher o sofrimento e o adoecimento psíquico no seu processo inicial, acolhendo as necessidades dos usuários, em um momento no qual precisamos apostar em trocas afetivas, em processos terapêuticos que possam ressignificar o sofrimento, a tristeza, a desesperança e o adoecimento ${ }^{14}$.

Uma das práticas utilizadas pelos profissionais das equipes intermediárias da SMAPS são as oficinas terapêuticas, que, por sua vez, auxiliam as pessoas no sentimento de pertencimento a uma comunidade, permitindo ampliar as formas de comunicação dos sentimentos 
de agressividade e sofrimento no momento em que as mãos trabalham e os profissionais fazem as intervenções singulares. A oficina terapêutica mostra-se um espaço em que as pessoas podem expressar seus sofrimentos e reações, permitindo uma reflexão para além do consultório psicológico. Elas funcionam como um dos elementos organizadores do cotidiano. São estratégias de cuidado, interação e socialização.

Tendo em vista que as intervenções da SMAPS, na atenção primária, baseiam-se nas necessidades das pessoas afetadas, as quais são alteradas e ressignificadas ao longo do tempo, era notório que essas estratégias e os dispositivos criados para ampliar o cuidado para a população deveriam ter um planejamento de continuidade considerando essa complexidade. Nesse sentido, o novo modelo de organização da rede demandou meios de criar possibilidades de garantia da sustentabilidade desse modelo. Avaliando os primeiros seis meses pós-desastre, percebe-se que as demandas seguiam intensas e desafiadoras a cada nova etapa do processo.

É esperado que, após um grande desastre, as consequências clínicas na saúde da população apresentem piora, como nos casos em que a hipertensão não é controlada mais com a mesma dosagem do medicamento e a diabetes apresenta alteração que antes do evento não acontecia. $\mathrm{O}$ corpo tende a adoecer mais, e a rotina de espera por exames e cirurgias tem potencializado o sentimento de revolta. As dores psíquicas sobrecarregam os corpos antes já adoecidos, e a dor se transforma em uma proporção ao tamanho do vazio e desesperança.

Adoecimentos que já estavam em curso foram e seguem sendo agravados pela intensidade e amplitude do evento. Mostrou-se importante lidar com planejamentos e ações que fossem flexíveis e que deixassem espaço para novas produções de vida, estimulando as redes de apoio socioafetivo para que os arranjos assistenciais pudessem ser moldados a novas realidades.

\section{Considerações finais}

Tendo como base o acompanhamento da rede municipal de saúde nos primeiros seis meses subsequentes ao desastre da Vale S.A. em Brumadinho, mostraram-se muitos os desafios postos para que o SUS se readapte à nova conjuntura. Parte dessas dificuldades estiveram diretamente relacionadas com o sofrimento intenso pela amplitude das perdas socioafetivas vivenciadas, bem como pela perda de recursos financeiros e de emprego, pelas consequências ambientais ainda pouco mensuradas, pela demanda por ressignificação de uma identidade da comunidade residente. A tristeza, o medo e a insegurança da população mostraram-se eminentes, sendo objeto de cuidado das equipes de atenção primária, da SMAPS e das práticas integrativas e complementares, bem como da rede ampliada de cuidado.

A fim de contemplar parte dessa demanda emergente de um novo perfil da população, iniciou-se o desenvolvimento de uma pesquisa de coorte para avaliar os impactos desse desastre na saúde da população, sendo esta desenvolvida por uma equipe de pesquisadores nacionais sob a coordenação da Fundação Oswaldo Cruz de Minas Gerais, e o financiamento do Ministério da Saúde. Ressalta-se que tal pesquisa tem como um dos eixos específicos a SMAPS pós-desastre em Brumadinho.

Ainda no que concerne à pesquisa coorte, prevê-se que no decurso dos próximos quatro anos busque-se compreender as mudanças na dinâmica de vida da população de Brumadinho, apontando fragilidades e potencialidades; e possibilitando que o poder público possa repensar as políticas públicas locais de acordo com as novas necessidades da população. A partir de ações como o acompanhamento a médio e longo prazo, espera-se que seja possível auxiliar a população na fase de reconstrução pós-desastre, auxiliando os atores locais na tomada de decisão e a população com as ações de cuidado direto.

Entendemos que são muitos os desafios para construir de forma integrada, nas diferentes 
áreas e nas esferas de gestão, estratégias de fortalecimento, ações de cuidado, medidas de vigilância e ofertas para garantia do acesso e cuidado integral. Um trabalho articulado entre o SUS e os diferentes setores, como a educação e a assistência social, mostrou-se importante e primordial, apontando para a necessidade de continuidade desses pontos na agenda do SUS.

\section{Referências}

1. Instituto Brasileiro de Geografia e Estatística. Panorama [internet]. [acesso em 2019 set 29]. Disponível em: https://cidades.ibge.gov.br/brasil/mg/brumadinho/panorama.

2. Defesa Civil Estadual de Minas Gerais. Informações: desastre barragem de rejeitos de Brumadinho [internet]. [acesso em 2019 set 29]. Disponível em: http:// www.defesacivil.mg.gov.br/index.php?option=com content\&view $=$ article\&id $=7$.

3. Franco MHP. Crises e desastres: a resposta psicológica diante do luto. O Mundo da Saúde [internet]. 2012 [acesso em 2019 ago 14]; 36(1):54-58. Disponível em: http://bvsms.saude.gov.br/bvs/artigos/mundo_saude/crises_desastres_resposta_psicologica_luto.pdf.

4. Companhia Vale do Rio Doce. Indenizações individuais ou por grupo familiar [internet]. [acesso em 2019 mar 25]. Disponível em: http://vale.com/brasil/

\section{Colaboradoras}

Noal DS (0000-0003-3970-6783)*, Braga VMR (0000-0002-8367-0637)*, Leal MB (0000-0002-1453-6100)*, Vargas AR (00000003-3354-9094)* e Eliazar P (0000-00028135-4294)* contribuíram igualmente para a elaboração do manuscrito.
${ }^{*}$ Orcid (Open Researcher and Contributor ID). pt/aboutvale/servicos-para-comunidade/minas-gerais/atualizacoes_brumadinho/paginas/indenizacoes.aspx.

5. Brumadinho. Secretaria Municipal de Saúde. Atendimentos das Equipes Intermediárias em Saúde Mental [internet]. [acesso em 2019 set 29]. Disponível em: https://brumadinho.mg.gov.br/.

6. Instituto Inhotim. Histórico [internet]. [acesso em 2019 set 29]. Disponível em: https://inhotim.org.br/.

7. Organización Panamericana de la Salud. Guía práctica de salud mental en situaciones de desastres [internet]. Washington, DC: Opas; 2009. [acesso em 2019 set 29]. Disponível em http://wwwl.paho.org/hq/ dmdocuments/2008/GuiaPracticadeSaludMental. pdf?ua=1.

8. Inter-Agency Standing Committee. Guidelines on 
mental health and psychosocial support in emergency settings. Geneva: Inter-Agency Standing Committee; 2007.

9. dation; Visão Global Internacional. Primeiros cuidados psicológicos: guia para trabalhadores de campo. Geneva: OMS; 2015.

10. Organización Mundial de la Salud; Alto Comissariado das Nações Unidas para os Refugiados - ACNUR. Evaluación de necesidades y recursos psicosociales y de salud mental [internet]. 2015. [acesso em 2019 set 29]. Disponível em: https://apps.who.int/iris/ bitstream/handle/10665/159202/9789243548531_ spa.pdf;sequence $=2$.

11. Brasil. Ministério da Saúde. Nota Técnica n ${ }^{\circ} 11 / 2019$ -CGMAD/DAPES; SAS; MS que esclarece sobre mudanças na Política Nacional de Saúde Mental e nas Diretrizes da Política Nacional sobre Drogas [internet]. [acesso em 2019 set 29]. Disponível em: http://pbpd. org.br/wp-content/uploads/2019/02/0656ad6e.pdf.

12. Tanaka OY, Ribeiro EL. Ações de Saúde Mental na Atenção Básica: Caminho para Ampliação da Integralidade da Atenção. Ciênc. Saúde Colet. [internet].
2009 [acesso em 2019 set 29]; 14(2):477-486. Disponível em: https://www.scielosp.org/pdf/csc/2009. v14n2/477-486/pt.

13. Souza AC, Rivera FJ. A inclusão das ações de saúde mental na Atenção Básica: ampliando possibilidades no campo da saúde mental. Revista Temp. Actas Saúde Colet. [internet]. 2010 [acesso em 2019 set 29]; 4(1):105-14. Disponível em: http://www6.ensp. fiocruz.br/repositorio/sites/default/files/arquivos/ Inclus\%C3\%A3o.pdf.

14. Noal DS, Rabelo IVM, Chachamovich E. O impacto na saúde mental dos afetados após o rompimento da barragem da Vale. Cad. Saúde Pública [internet]. 2019 [acesso em 2019 set 23]; 35(5):e00048419. Disponível em: https://doi.org/10.1590/0102-311X00048419.

15. Brasil. Ministério da Saúde. Portaria $\mathrm{n}^{0} 971$ de 3 de maio de 2006. Aprova a Política Nacional de Práticas Integrativas e Complementares. Brasília, DF: Diário Oficial da União. 4 Maio 2006.

Recebido em 30/09/2019

Aprovado em 04/02/2020

Conflito de interesses: inexistente

Suporte financeiro: não houve 